\title{
Prenatal diagnosis of chromosomal abnormalities using array-based comparative genomic hybridization
}

Trilochan Sahoo, MD, Sau Wai Cheung, PhD, Patricia Ward, MS, Sandra Darilek, MS, Ankita Patel, PhD, Daniela del Gaudio, PhD, Sung Hae L. Kang, PhD, Seema R. Lalani, MD, Jiangzhen Li, MD, Sallie McAdoo, MS, Audrey Burke, MS, Chad A. Shaw, PhD, Pawel Stankiewicz, MD, PhD, A. Craig Chinault, PhD, Ignatia B. Van den Veyver, MD, Benjamin B. Roa, PhD, Arthur L. Beaudet, MD, and Christine M. Eng, MD

\begin{abstract}
Purpose: This study was designed to evaluate the feasibility of using a targeted array-CGH strategy for prenatal diagnosis of genomic imbalances in a clinical setting of current pregnancies. Methods: Women undergoing prenatal diagnosis were counseled and offered array-CGH (BCM V4.0) in addition to routine chromosome analysis. Array-CGH was performed with DNA directly from amniotic fluid cells with whole genome amplification, on chorionic villus samples with amplification as necessary, and on cultured cells without amplification. Results: Ninety-eight pregnancies (56 amniotic fluid and 42 CVS specimens) were studied with complete concordance between karyotype and array results, including 5 positive cases with chromosomal abnormalities. There was complete concordance of array results for direct and cultured cell analysis in 57 cases tested by both methods. In 12 cases, the array detected copy number variation requiring testing of parental samples for optimal interpretation. Array-CGH results were available in an average of 6 and 16 days for direct and cultured cells, respectively. Patient acceptance of array-CGH testing was $74 \%$. Conclusion: This study demonstrates the feasibility of using array-CGH for prenatal diagnosis, including reliance on direct analysis without culturing cells. Use of array-CGH should increase the detection of abnormalities relative to the risk, and is an option for an enhanced level of screening for chromosomal abnormalities in high risk pregnancies. Genet Med 2006:8(11):719-727.
\end{abstract}

Key Words: comparative genomic hybridization, prenatal diagnosis, genetic counseling, microdeletion syndromes

Prenatal cytogenetic testing is an option for pregnancies at increased risk of chromosomal abnormalities based on maternal age, abnormal maternal serum screening results, or fetal anomalies observed by ultrasound. Moreover, the risk-to-benefit ratio of offering invasive prenatal testing to all pregnancies regardless of risk factors is an active area of discussion in the obstetrics and genetics communities. ${ }^{1-3}$ Conventional forms of cytogenetic analysis such as G-banded chromosome analysis and rapid FISH (fluorescence in situ hybridization) techniques are currently the mainstay of prenatal diagnosis of chromosomal abnormalities. ${ }^{4-7}$ Conventional karyotyping on prenatal cells yields lower band resolution than on blood cells, making detection of subtle abnormalities more difficult, and numerous common microdeletion syndromes are not detectable by even blood karyotype. Although phenotypic features of microdeletion or duplication syndromes may direct the use of

From the Department of Molecular and Human Genetics Baylor College of Medicine, Houston, TX. Christine M. Eng, MD, Department of Molecular and Human Genetics, Baylor College of Medicine, NAB 2015 Houston, Texas 77030.

Submitted for publication May 18, 2006.

Accepted for publication August 10, 2006.

DOI: 10.1097/01.gim.0000245576.47154.63 syndrome-specific FISH tests in the postnatal period, there is limited potential for targeted testing in the prenatal period. Furthermore, such genomic disorders are often sporadic events resulting from de novo rearrangements. The current guidelines and standards for prenatal diagnosis of chromosomal defects predate the technical feasibility for detection of an increasing number of chromosomal deletion and duplication syndromes spread throughout the genome by methods such as array-based comparative genomic hybridization (array-CGH; also known as chromosomal microarray analysis or CMA). ${ }^{8}$

Microarrays using large-insert clones can detect deletions or duplications that are tens to hundreds of $\mathrm{kb}$ in size, which is well below the level of discrimination for G-banded karyotype analysis. ${ }^{9-11}$ Moreover, array-CGH offers rapid, high-throughput analysis on minimal amounts of DNA, two prerequisites for any platform applied to prenatal diagnosis. A comprehensive profile of clinically relevant genomic gains and losses that are associated with genomic disorders would therefore make array-CGH a potentially useful tool for prenatal cytogenetic analysis of high-risk pregnancies. In addition, the use of wholegenome amplification (WGA) techniques to amplify extremely small amounts of genomic DNA would enable analysis directly on amniotic fluid (AF) cells or chorionic villus samples (CVS) 
without culturing, leading to more rapid results without the added time or cost of cell culture. ${ }^{12,13}$

There are several reports of the use of array-CGH for multiplex diagnosis of cytogenetic abnormalities using blood samples and at least one report of the application of array-CGH to previously studied, archived prenatal samples, but to date, there has been no experience with the use of this technique to monitor and report on current pregnancies..$^{11,14-19}$ We designed a targeted microarray of 366 large genomic clones covering the 41 clinically relevant subtelomeric regions as well as genomic positions corresponding to 55 different genomic disorders, validated its use for molecular cytogenetic diagnosis on samples with known abnormalities, and completed over 1,500 clinical cases on referred blood samples. ${ }^{11}$ Based on this collective experience with array-CGH, we carried out a prospective study to assess the feasibility of offering array-CGH in the clinical prenatal diagnostic setting with informed consent and counseling, including reliance on direct analysis without culturing cells. We present here the results of a pilot study on 98 prenatal specimens from current pregnancies to compare results obtained by standard karyotype and array-CGH.

\section{METHODS}

\section{Subject recruitment}

The study was approved by the Institutional Review Board of Baylor College of Medicine. The description of the availability of array-CGH was initially communicated by the referring physician to couples considering an invasive prenatal procedure; if the couple expressed interest in participating in the study, they were referred to the study genetic counselor or medical geneticist who obtained written informed consent. Couples who chose to have amniocentesis or chorionic villus sampling were offered the option of array-CGH in addition to karyotype. The indications for testing included increased risk for fetal aneuploidy associated with advanced maternal age and/or abnormal serum screening results, risk for a cytogenetic abnormality based on family history, and abnormal ultrasound findings for which a diagnosis was being sought.

\section{Pretest counseling and informed consent}

The pretest genetic counseling consisted of a standard genetic counseling session with recording of past medical and obstetric history, a pedigree with family medical history, the patient's history of exposure to infectious agents or potential teratogens, and an overall estimation of fetal risk. In addition, the array-CGH technique and study protocol were explained in detail including the risks and benefits of participation. The array-CGH analysis was offered at no additional fee.

\section{Specimen preparation}

The amniotic fluid (AF) and chorionic villus samples (CVS) were obtained by the subjects' obstetricians using their standard clinical procedures. Blood samples were required for maternal cell contamination studies and were requested from both parents for analysis of familial variants (if warranted by
array-CGH results). All AF and CVS samples were prepared for standard G-band karyotype per clinical laboratory protocols. The remainder of the fetal sample was prepared for array-CGH analysis using direct and/or cultured cells.

DNA extraction was performed utilizing the Puregene DNA Purification kit according to the manufacturer's protocols (Gentra Systems, Minneapolis, MN). If adequate amounts of fetal material were obtained (a minimum of $5 \mathrm{mg}$ CVS tissue or $5 \mathrm{~mL}$ of $\mathrm{AF}$ ), the fetal sample was prepared for two independent array-CGH analyses using both direct genomic DNA and DNA obtained from cultured cells. For all amniotic fluid samples and for those CVS samples with less than the minimum amount, $10 \mathrm{ng}$ of direct genomic fetal DNA sample was subjected to whole genome amplification (WGA) according to standard procedures (GenomePlex WGA kit, SIGMA, St. Louis, $\mathrm{MO}$ ). Immediately upon receipt of a sample, the sex of the fetus was determined by PCR and the test DNA was hybridized to control DNA of the same sex. Reference genomic DNA was derived from peripheral blood of phenotypically normal male and female control individuals. When WGA products were used, reference DNAs were amplified in parallel with the test DNA. DNA from both direct and cultured samples was tested for maternal cell contamination using the PCR-based Identifiler system (AmpFLSTR, Applied Biosystem, Foster City, CA).

Prior to the start of the analysis of ongoing pregnancies, studies to validate the array-CGH method on DNA isolated from amniocytes or CVS were performed on known abnormal cases. In addition, parallel testing by array-CGH was performed on native genomic DNA and WGA-amplified DNA isolated from whole blood specimens from cases with known abnormalities.

\section{Microarray constitution, hybridization and data analysis}

The microarrays were manufactured using protocols described previously. ${ }^{16}$ Briefly, BAC DNA was prepared using standard alkaline lysis methods and chemically modified for array printing as described. Chemically activated DNA [using (3-glycidoxypropyl) trimethoxysilane; Sigma, St. Louis, MO)] was printed onto alkaline/acid cleaned, Bis(trichlorosily1)octane-treated (Gelest Inc. Morrisville, PA) glass slides using an Omnigrid Accent printer (Genomic Solutions, Ann Arbor, MI). All clones were printed in duplicate from a single 384 well plate in spatially distinct subarrays.

The Baylor College of Medicine Chromosome Microarray version 4.0 (BCM V4.0) was used for this study (www.bcm. edu/cma/table.htm, Table 3). This array was designed to provide redundancy with high sensitivity and specificity for detection of well-characterized disorders, while minimizing the detection of variations of uncertain clinical significance. The array was comprised of 3 to $10 \mathrm{BAC}$ or PAC clones per disease or subtelomeric locus. A total of 366 distinct clones, including 200 clones covering the 41 clinically relevant subtelomeric regions and 166 clones from genomic positions corresponding to 55 different genetic disorders were included in version 4.0. The clones were selected from NCBI/UCSC public databases or from the FISH probe panel routinely used in the cytogenetic laboratory. Chromosomal positions detected by all clones were 
FISH-verified before use on the microarray and any clones that hybridized to multiple chromosomal locations were excluded. Versions of the array are periodically updated to add clones of clinical significance and remove clones found to be highly polymorphic.

DNA labeling and hybridizations were performed as described. ${ }^{11,20}$ For each patient sample, two experiments were performed with reversal of the dye labels for the control and test samples, followed by integration of the data from both dye-reversed hybridizations to determine inferences for each case (Fig. 1). Microarray image files were quantified using GenePix Pro 5 software. The quantitation data were subjected to normalization as described previously, and the dye-reversed data were combined to determine a single fold-change value for each clone. ${ }^{11,20}$ Inferences were made for all clones using these final combined data values (Fig. 1). ${ }^{11,20}$ All analyses were performed on $\log _{2}$ ratios using code for the normalization and inference that was implemented in the $\mathrm{R}$ statistical programming language.

The results of the array-CGH analyses were communicated to patients through their physician or genetic counselor. Pregnancies with abnormal results were counseled in person by their physician or a medical geneticist and genetic counselor. Cases in which copy number variants (CNV) were detected were referred to an internal interdisciplinary panel consisting of medical geneticists, maternal fetal medicine specialists, and cytogeneticists who reviewed the data and reached consensus regarding the interpretation prior to release of the results.

\section{FISH analysis}

Metaphase spreads for detection of deletions were prepared from amniocytes or CVS using standard procedures. Interphase nuclei were analyzed for duplications/copy number gains. Miniprep BAC DNA (100 ng) was labeled with Spectrum Orange-dUTP or Spectrum Green-dUTP (Vysis, Downers Grove, IL) according to manufacturer's protocol and used as probes for FISH analysis using standard protocols. ${ }^{7}$

\section{RESULTS}

\section{Validation of array-CGH and whole genome amplification protocols}

An initial validation of the array-CGH tool using DNA from 12 cytogenetically abnormal AF or CVS cultures was carried out before initiating the prospective study. These cases included the following abnormalities: 47,XXY; 47,XX, +18; 47,XY,+13; 47,XY,+21; 69,XXX; 46,XX, del(X)(p22.31); and $47, \mathrm{XX},+\mathrm{i} 12(\mathrm{p} 10)$. All segmental and whole-chromosome aneuploidies were accurately identified (data not shown). Additionally, the feasibility of using WGA to amplify nanogram amounts of genomic DNA was tested with six postnatal samples with known microdeletions or duplications including del11(p13), del20(p12), dup17(p12), del8(q24.1). Array-CGH profiles using DNA after WGA reproduced results that were completely concordant with those from native genomic DNA (e.g., Fig. 1B), with no detectable amplification bias.

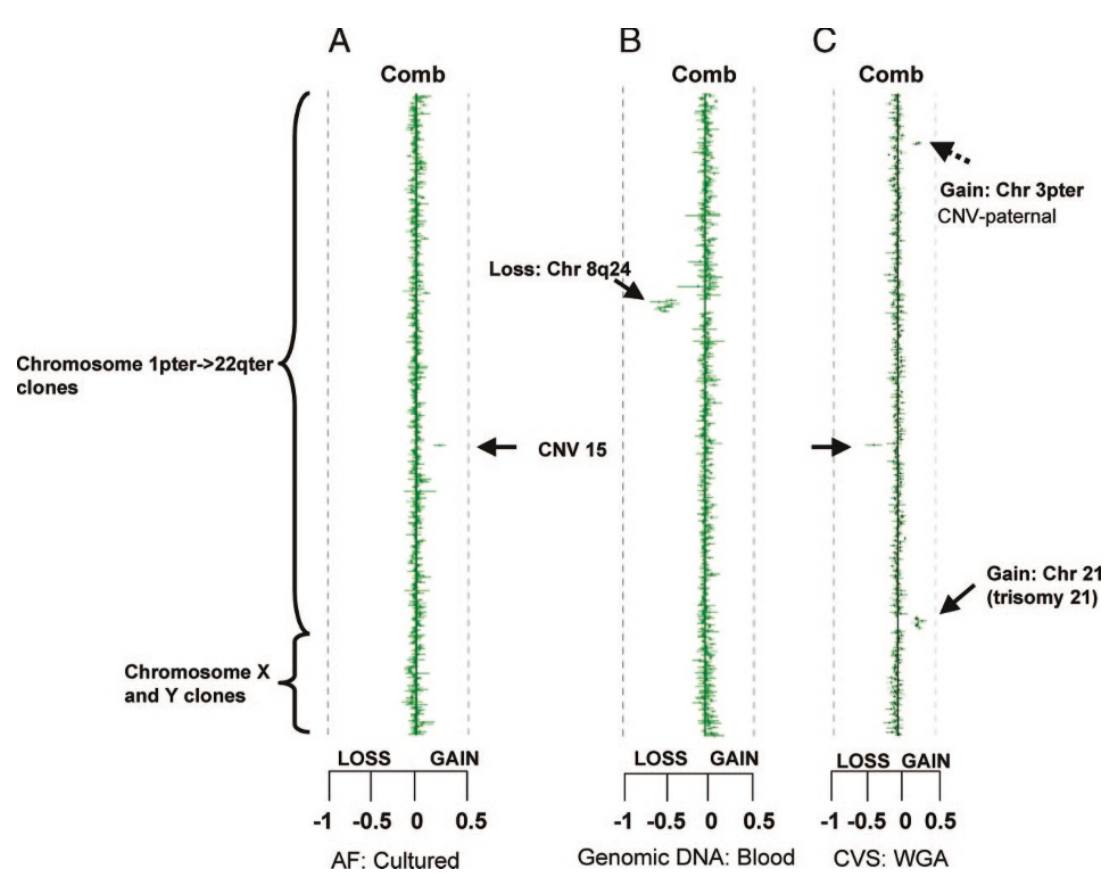

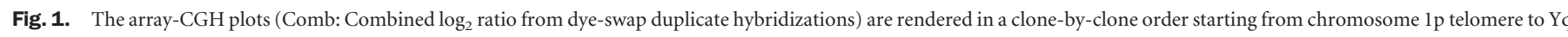

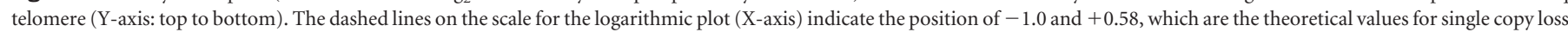

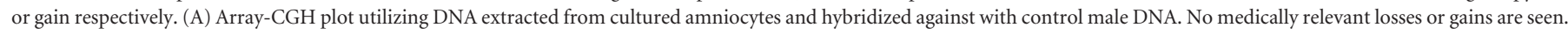

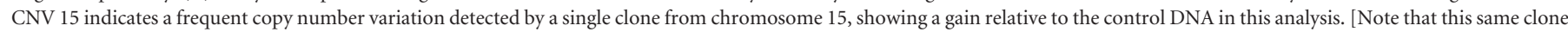

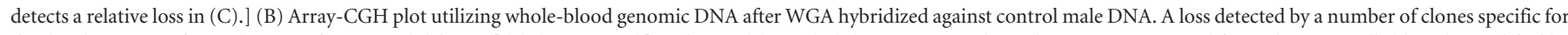

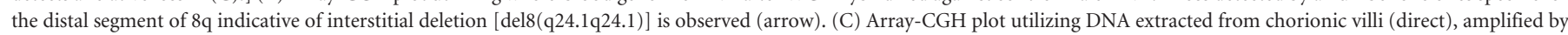

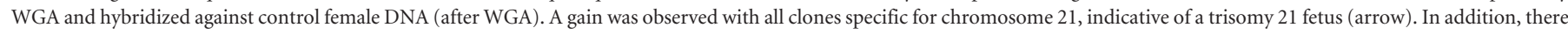
is a gain detected by 2 clones on $3 p$ that was inherited from the phenotypically normal father and was interpreted as a benign familial copy number variant (CNV). 
Table 1

Sample distribution including indications for prenatal diagnosis

\begin{tabular}{lcccc}
\hline & \multicolumn{2}{c}{ Indication } \\
\cline { 2 - 5 } Sample Type & AMA $^{a}$ & Abnormal U/S & Abnormal Serum Screen & Previous Abnormal Fetus \\
\hline Amniotic fluid $(\mathrm{N}=56)$ & 31 & $16(2$ AMA $)$ & $4(1$ AMA) & 5 \\
Chorionic villi $(\mathrm{N}=42)$ & 33 (1 with balanced translocation) & 3 (all AMA) & 0 & 6 \\
\hline
\end{tabular}

${ }^{a}$ AMA, advanced maternal age.

${ }^{b} \mathrm{U} / \mathrm{S}$, ultrasound.

\section{Prenatal study population}

After informed consent, 98 couples chose to have arrayCGH analysis in addition to a standard karyotype for their current pregnancies. As shown in Table 1, 56 (57\%) analyzed samples were amniotic fluid and 42 (43\%) were CVS. The indications for prenatal testing were advanced maternal age (64; $65 \%)$, fetal ultrasound anomalies $(19 ; 19 \%)$, previous history of a child with anomalies $(11 ; 11 \%)$, and abnormal maternal serum screening result $(4 ; 4 \%)$.

\section{G-Banded karyotype}

Conventional G-banding and karyotype analysis were conducted on all 98 cases. These analyses detected 4 cases of trisomy 21 and 1 unbalanced translocation of chromosomes 3 and 7. No other abnormalities were detected. The average reporting time for karyotype on these 98 cases was 10 days from the time of the prenatal procedure.

\section{Array-CGH analysis}

The array-CGH analysis was performed concurrently with the karyotype in a blinded fashion. For all 98 cases, analysis was performed on DNA isolated from cultured amniocytes or chorionic villi. The array-CGH analyses detected all 4 cases of trisomy 21 and the case with an unbalanced translocation between chromosomes 3 and 7 , demonstrating complete concordance with the results of karyotype analysis. For the latter case, array-CGH detected a gain of copy number with 3 clones on chromosome region $3 \mathrm{q}$ and a loss of copy number with 8 clones on chromosome region $7 q 36.3$ that included the sonic hedgehog homolog gene $(\mathrm{SSH})$, which causes holoprosencephaly-3 (Fig. 2). These findings were consistent with the fetal ultrasound finding of holoprosencephaly. Adequate coverage of the above-mentioned genomic segments revealed the unbalanced rearrangement and facilitated an accurate estimate of the imbalance. The same cannot be predicted for genomic regions with minimal or no coverage, an issue that may be addressed by inclusion of genomic clones at regular intervals across the entire genome.

After the first 40 samples, the protocol was modified to include analysis of AF cells and CVS tissue directly without cell culture. In the last 58 cases (26 AF and $32 \mathrm{CVS}$ ), a duplicate array-CGH analysis was performed on DNA extracted directly from the AF cells with WGA or from CVS with (21/32) or without (11/32) WGA. The results from both sources were in complete agreement. The average reporting time of arrayCGH was 6 days from the time of the prenatal procedure for analysis of direct CVS or amniocytes and 16 days for cultured CVS and AF.

\section{Copy number variants (CNV)}

In 30 cases, array-CGH detected a gain or loss of copy number in a fetus with a clone (or clones) which was known to show a frequent normal variation in copy number based on prior or concurrent analysis of hundreds of blood samples. These cases
A

\begin{tabular}{|l|l|l|}
\hline Clone & Location & Ratio \\
\hline RP11-17P4 & $3: q 29$ & 0.295 \\
\hline RP11-1D19 & $3: q 29$ & 0.276 \\
\hline RP11-432D10 & $3: q 29$ & 0.353 \\
\hline RP5-1015024 & $7: q 36.3$ & -0.628 \\
\hline RP11-6903 & $7: q 36.3$ & -0.447 \\
\hline RP11-52501 & $7: q 36.3$ & -0.653 \\
\hline RP4-782K24 & $7: q 36.3$ & -0.197 \\
\hline RP11-11B21 & $7: q 36.3$ & -0.231 \\
\hline RP11-324E12 & $7: q 36.3$ & -0.167 \\
\hline RP4-764012 & 7:q36.3 & -0.422 \\
\hline GS-3K23 & 7:qter & -0.463 \\
\hline
\end{tabular}

B

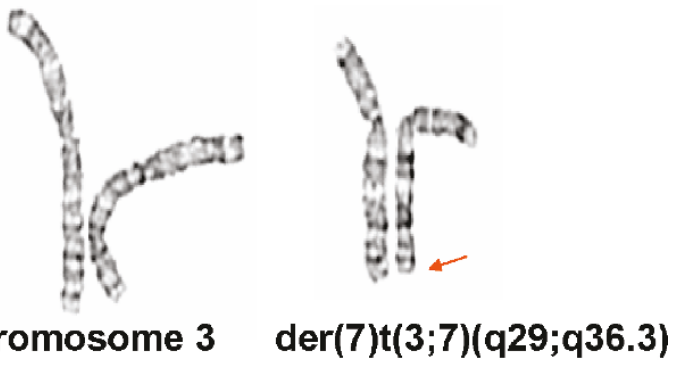

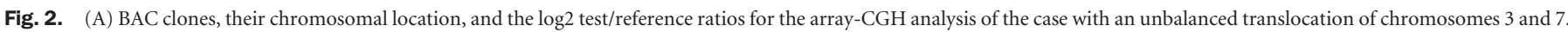

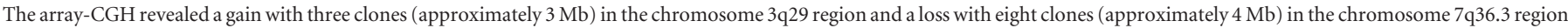
extending distally. (B) G-banded karyotype of the same case showing a normal chromosome 3 and the derivative chromosome 7 (arrow). 
Table 2

Prenatal cases with copy number variants requiring additional analyses

\begin{tabular}{|c|c|c|c|c|}
\hline Case & Array Finding & FISH & Parents & Comments \\
\hline 1 & Gain 3 clones 15q11.2 & Detected by FISH & Present in mother & Likely benign \\
\hline 2 & Gain 3 clones STS region & Inconclusive & Not available & Seen in other families; likely benign \\
\hline 3 & Gain 2 clones $18 \mathrm{p}$ & Detected by FISH & Not present in either parent & $\begin{array}{l}\text { Not present in liveborn; died pentalogy Cantrell; artifact } \\
\text { or low level mosaicism }\end{array}$ \\
\hline 4 & Gain 1 clone Xp & Not detected & Not present in mother & Infant healthy at birth; possibly de novo $\mathrm{CNV}$ \\
\hline 5 & Gain 2 clones $6 \mathrm{q}$ & Not done & Present in father & Likely benign \\
\hline 6 & Gain 3 clones $2 \mathrm{q}$ & Detected by FISH & Present in father & Seen in other patients postnatal as $\mathrm{FCNV}^{a}$; Likely benign \\
\hline 7 & Gain of 3 clones on $3 p$ & Not done & Present in father & Trisomy 21 (case in Fig 1C); CNV likely benign \\
\hline 8 & Loss 1 clone $2 p$ & Not detected & Present in father & Likely benign \\
\hline 9 & Gain 1 clone $19 q$ & Not done & Present in mother & Likely benign \\
\hline 10 & Gain 1 clone 10q & Not done & Present in mother & Likely benign \\
\hline 11 & Gain 1 clone $\mathrm{Xp}$ & Not done & Present in father & Likely benign \\
\hline 12 & Gain 1 clone $16 p$ & Not done & Present in father & Likely benign \\
\hline
\end{tabular}

${ }^{a}$ FCNV, familial copy number variant.

were interpreted as normal without analyzing the parents. In 12 cases (Table 2), array-CGH analysis revealed losses or gains with 1 or more clones that necessitated further analysis because the pattern of gain or loss was not consistent with a known genetic syndrome, or detection of gain or loss was infrequent for the clones involved. Prompt parental analysis was offered to these families. The extended analysis for each of these cases included FISH and/or array analysis of the family members as appropriate. It should be noted that the variation involving three clones at 15q11q13 in case 1 involves clones containing segmental duplications known to result in copy number losses or gains when tested by array-CGH. Similarly, the genomic sequence content for variant clones in cases 2, 6 and 7 revealed the presence of LCRs that contribute to clone behavior (loss or gain compared to control DNA) on array-CGH. In 9/12 cases, the gain or loss was detectable in a parent by array-CGH and/or FISH. In 1/12 cases, there was a gain detected with 3 clones in the steroid sulfatase region of the X chromosome. Following genetic counseling, the parents chose not to be studied. This gain has been detected in apparently benign circumstances previously (i.e., present in a child as well as a phenotypically normal parent). In another case, a male fetus was found to have a gain detected with one clone on Xp that was not detectable by FISH and was not found in the mother. For these two cases, the post-test counseling consisted of full discussion of the arrayCGH results as well as the lack of definitive data in our experience or in the literature to assign a significant clinical risk. Both pregnancies continued, both children were born at term, had no physical anomalies, and are doing well. In another case, an apparent gain of copy number on chromosome segment $18 \mathrm{p} 11.3$ detected with one clone was seen in an early case where only cultured cells were analyzed. The finding was confirmed by FISH, and was not present in the parents. A fetal ultrasound showed an abdominal wall defect and a fetal MRI was consis- tent with pentalogy of Cantrell. The infant died within the first month of life. Array analysis on postnatal tissue did not detect the gain. This case may represent low level mosaicism in the original sample for a duplication of $18 \mathrm{p} 11.3$ or an artifact arising in tissue culture. Interestingly, pentalogy of Cantrell has been reported in a case of trisomy 18. ${ }^{21}$ Thus, in our series, 2/98 cases had possible de novo abnormalities; 1 showed an apparent gain of copy number with a clone on Xp in the fetal sample, but this change was not detectable by FISH, and was not present in the mother. The second case likely represented low level mosaicism or tissue culture artifact. More recent experience from our group indicates that mosaicism at a level $>7 \%$ can be detected by array-CGH.

\section{Counseling and patient decision-making}

For a subset of 53 couples who received genetic counseling by a single genetic counselor, the data were examined in more detail to discern trends in test acceptance. These 53 couples were at increased risk because of advanced maternal age, abnormal fetal ultrasound findings, abnormal serum screening, or a family history of a previous child with anomalies. Following the initial counseling, $45 / 53$ couples ( $85 \%$ ) chose to have amniocentesis or CVS performed on the pregnancy. Of these 45 couples, 33 (73\%) chose to have array-CGH testing in addition to the standard karyotype. The most common reasons for accepting array-CGH testing included the detection of fetal anomalies in the current pregnancy, a history of anomalies in a previous child, and the desire for more comprehensive testing on their prenatal sample. Frequent reasons for declining testing included the risk of increased anxiety while waiting for the results of the additional testing, their perception that the disorders tested on the microarray were rare, and their uncertainty over accepting a new test. 
Table 3

Abnormalities detected by the array-CGH platform (BCM version 4$)^{a, b}$

\begin{tabular}{|c|c|c|c|c|}
\hline OMIM\# & Loci & $\begin{array}{c}\text { Abnormalities Detected by CMA (Subtelomeric deletion/ } \\
\text { duplication for all chromosome arms) }\end{array}$ & $\begin{array}{l}\text { Cytogenetic Abnormalities } \\
\text { (All subtelomeres excluding the } \\
\text { short arm of the acrocentric } \\
\text { chromosomes }\end{array}$ & Genes \\
\hline & & Aneuploidy for chromosomes $13,18,21, \mathrm{X}$, and $\mathrm{Y}$ & & \\
\hline & $1 \mathrm{p} 36$ & 1p36 deletion and/or duplication & $1 \mathrm{p} 36$ deletion and/or duplication & \\
\hline 256100 & NPH1 & Nephronophthisis & $2 \mathrm{q} 13$ homozygous deletion & NPHP1 \\
\hline 194190 & WHS & Wolf-Hirschhorn syndrome & $4 \mathrm{p} 16.3$ deletion & \\
\hline 180500 & RIEG1 & Rieger syndrome & $4 \mathrm{q} 25$ deletion & PITX2 \\
\hline 123450 & CdCS & Cri-du-Chat syndrome & $5 \mathrm{p} 15.2$ deletion & \\
\hline$>122470$ & CdLS & Cornelia de Lange syndrome & 5 p13.2 deletion & $N I P B L$ \\
\hline 117550 & SOS & Sotos syndrome & $5 q 35$ deletion & NSD1 \\
\hline 119600 & $\mathrm{CCD}$ & Cleidocranial dysplasia & $6 \mathrm{p} 21.1$ deletion & RUNX2 \\
\hline 101400 & SCS & Saethre-Chotzen syndrome & $7 \mathrm{p} 21.1$ deletion & TWIST \\
\hline 175700 & GCPS & Greig cephalopolysyndactyly syndrome & 7 p13 deletion & GLI3 \\
\hline 194050 & WBS & Williams-Beuren syndrome & 7q11.2 deletion & $E L N$ \\
\hline \multirow[t]{2}{*}{142945} & HPE3 & Holoprosencephaly 3 & 7q36.3 deletion & SHH \\
\hline & & 8 p22 deletion/duplication syndrome & 8p22-p23.1 duplication/deletion & \\
\hline 150230 & LGS & Langer-Giedion syndrome & $8 \mathrm{q} 24.11-\mathrm{q} 24.13$ deletion & TRPS1 and/or EXT1 \\
\hline 190350 & TRPS1 & Trichorhinophalangeal syndrome,Type I & $8 \mathrm{q} 24.12$ deletion & TRPS1 \\
\hline 146255 & HDR & $\begin{array}{l}\text { Hypoparathyroidism, sensorineural deafness and renal } \\
\text { dysplasia }\end{array}$ & 10p14-p15.1 deletion & GATA3 \\
\hline 601362 & DGS2 & DiGeorge syndrome 2 & 10p13-p14 deletion & \\
\hline 608071 & SHFM3 & Split hand/split foot syndrome 3 & 10q24 duplication & SHSF3 \\
\hline 130650 & BWS & Beckwith-Wiedemann syndrome (BWS) & 11p15.5 duplication & IGF2 \\
\hline 106210 & AN2 & Aniridia type 2 & 11 p13 deletion & PAX6 \\
\hline 607102 & WT1 & Wilm's tumor 1 gene & 11 p13 deletion & $W T 1$ \\
\hline 194072 & WAGR & $\begin{array}{l}\text { Wilm's tumor-aniridia-genitourinary abnormalities } \\
\text { (WAGR) syndrome }\end{array}$ & $11 \mathrm{p} 13$ deletion & WT1/PAX6 \\
\hline 601224 & PSS & Potocki-Shaffer syndrome & $11 \mathrm{p} 11.2$ deletion & ALX4 and/or EXT2 \\
\hline 147791 & JBS & Jacobsen syndrome & $11 \mathrm{q} 24.3$ deletion & \\
\hline 180200 & RB1 & Retinoblastoma & 13q14 deletion & $R B 1$ \\
\hline 603073 & HPE5 & Holoprosencephaly 5 & $13 q 32.3$ deletion & ZIC2 \\
\hline 176270 & PWS & Prader Willi syndrome (PWS) & Paternal 15q11.2q13 deletion & SNRPN \\
\hline 105830 & AS & Angelman syndrome (AS) & Maternal 15q11.2q13 deletion & $U B E 3 A$ \\
\hline 209850 & & Autism & Maternal duplication $15 \mathrm{q} 11.2 \mathrm{q} 13$ & \\
\hline 191092 & TS 2 & Tuberous Sclerosis 2 & 16p13.3 deletion & TSC2 \\
\hline 180849 & RSTS & Rubinstein-Taybi syndrome & 16p13.3 deletion & CREBBP \\
\hline 600273 & PKDTS & Polycystic kidney disease/tuberous sclerosis & 16p13.3 deletion & PKD1 and/or TSC2 \\
\hline 247200 & MDLS & Miller-Dieker lissencephaly syndrome & 17p13.3 deletion & $\begin{array}{l}\text { LIS1 and/or } \\
\text { YWHAE }\end{array}$ \\
\hline 118220 & CMT1A & Charcot-Marie-Tooth disease type 1A & 17p12 duplication & PMP22 \\
\hline 162500 & HNPP & Hereditary neuropathy with liability to pressure palsies & 17 p12 deletion & PMP22 \\
\hline 182290 & SMS & $\begin{array}{l}\text { Smith-Magenis syndrome dup(17)(p11.2p11.2) } \\
\text { syndrome }\end{array}$ & $17 \mathrm{p} 11.2$ deletion & RAII \\
\hline 162200 & NF1 & Neurofibromatosis 1 & 17q11.2 deletion & NF1 \\
\hline
\end{tabular}


Table 3

Continued

\begin{tabular}{|c|c|c|c|c|}
\hline OMIM\# & Loci & $\begin{array}{c}\text { Abnormalities Detected by CMA (Subtelomeric deletion/ } \\
\text { duplication for all chromosome arms) }\end{array}$ & $\begin{array}{c}\text { Cytogenetic Abnormalities } \\
\text { (All subtelomeres excluding the } \\
\text { short arm of the acrocentric } \\
\text { chromosomes }\end{array}$ & Genes \\
\hline 114290 & CMPD & Campomelic dysplasia & $17 \mathrm{q} 24.3$ deletion & SOX9 \\
\hline 142946 & HPE4 & Holoprosencephaly 4 & 18 p11.31 deletion & TGIF \\
\hline 118450 & AGS & Alagille syndrome & 20p12 deletion & $J A G 1$ \\
\hline 190685 & DS & Down syndrome critical region & 21q22 duplication & \\
\hline 115470 & CES & Cat eye syndrome & $\operatorname{inv} \operatorname{dup}(22 \mathrm{q} 11.2)$ & \\
\hline 192430 & VCFS & Velocardiofacial syndrome & $22 \mathrm{q} 11.2$ deletion & $T B X 1$ \\
\hline 188400 & DGS1 & DiGeorge syndrome $1 \mathrm{dup}(22)(\mathrm{q} 11.2 \mathrm{q} 11.2)$ syndrome & 22q11.2 deletion & $T B X 1$ \\
\hline 127300 & LWD & Leri-Weill dyschondrosteosis & $\mathrm{Xp} 22.33 / \mathrm{Yp} 11.32$ & SHOX \\
\hline 308100 & STS & Steroid Sulfatase Deficiency & Xp22.32 deletion & STS \\
\hline 308700 & KMS & Kallmann syndrome 1 & Xp22.3 deletion & $K A L 1$ \\
\hline 309801 & MLS & Micophthalmia with Linear Skin Defects & Xp22.31 deletion & \\
\hline 300474 & GKD & Glycerol Kinase Deficiency & Xp22 deletion & $G K$ \\
\hline 300200 & $\mathrm{AHC}$ & Congenital adrenal hypoplasia & Xp21.3 deletion & NROB1 \\
\hline 300300 & BTK & Bruton agammaglobulinemia tyrosine kinase & $\mathrm{Xq} 22.1$ & BTK \\
\hline 312080 & PMD & Pelizaeus-Merzbacher disease & Xq22 duplication or deletion & $P L P 1$ \\
\hline 300123 & MRGH & Mental retardation X-linked & Xq27.1 deletion or duplication & SOX3 \\
\hline 312750 & RTT & Rett syndrome & Xq28 deletion & MECP2 \\
\hline 480000 & TDY & Testis-Determining Factor on $\mathrm{Y}$ & Yp11.2 deletion & $S R Y$ \\
\hline \multirow[t]{3}{*}{415000} & $\mathrm{AZFa}$ & Azospermia factor a & Yq11 deletion & \\
\hline & $\mathrm{AZFb}$ & Azospermia factor $b$ & Yq11 deletion & \\
\hline & $\mathrm{AZFc}$ & Azospermia factor $\mathrm{c}$ & Yq11.23 deletion & \\
\hline
\end{tabular}

${ }^{a}$ Detection rates by array-CGH for the conditions listed vary according to the proportion of cases related to microdeletion or microduplication of the relevant region.

${ }^{b} \mathrm{BCM}$ version 6.0 currently available.

\section{DISCUSSION}

The utility of array-CGH as a tool for identifying chromosomal imbalances in prenatal samples has been recently described ${ }^{17,18,22,23}$ but to our knowledge, this is the first study comparing the use of array-CGH to a standard karyotype for prenatal genetic diagnosis of a large spectrum of known genomic imbalances in ongoing pregnancies with results reported to participants. In a cohort of 98 cases, 4 cases of trisomy 21 were detected by both standard karyotypes and arrayCGH. A fetus carrying an unbalanced translocation inherited from a balanced translocation carrier parent was identified by both methods, but array-CGH demonstrated that the $7 \mathrm{q}$ breakpoint at band 36.3 involved a loss detected by 8 clones including the $\mathrm{SHH}$ gene (holoprosencephaly 3 ), consistent with the fetal finding of holoprosencephaly. No other abnormalities related to a known genetic disorder were detected by either method in this sample of 98 ongoing pregnancies.

For most cytogenetic abnormalities, array-CGH analysis is likely to be as effective as a standard karyotype in detecting gross aneuploidies, while having two important advantages:
The first is more reliable detection of subtle abnormalities that may be difficult to detect on prenatal samples (e.g., small telomeric gains or deletions); and the second is detection of abnormalities that are not detectable even by an optimal karyotype (e.g., cryptic telomeric deletions or syndromic deletions such as DiGeorge or Williams syndromes). These advantages substantially decrease the likelihood that a subtle abnormality will be undetected during prenatal diagnosis. In addition, the array analysis can be as rapid as, or more rapid than, karyotype when performed by direct analysis of AF cells or CVS. In terms of possible disadvantages, a noteworthy limitation is that the detection rate of individual syndromes by array-CGH is dependent on the cytogenetic and molecular cause of the syndrome. For example, a genetic disorder caused mainly by deletion mutations would have a high detection rate by array CGH, whereas a disorder caused mainly by point mutations and rarely by deletions, would have a lower detection rate by this method. Array-CGH using a focused array of the type used here could fail to detect a cytogenetically visible deletion in the middle of an arm of a chromosome. This problem could be 
addressed in the future using arrays with greater or even full genomic coverage. Also array-CGH would not detect triploidy of the whole genome with XXX but would indicate an abnormality for triploidy with XXY or XYY. The relative sensitivity of karyotype and array CGH for detecting mosaic abnormalities depends on the number of metaphases analyzed by karyotype and on the percentage of abnormal cells. Array-CGH will not detect balanced translocations or inversions. While their pathologic significance is rarely definitive in a prenatal setting, their detection provides important information for the family. For the present, the latter three of these limitations are compensated for by karyotype analysis. Therefore, we recommend that a concurrent karyotype be performed in addition to the microarray analysis for these reasons. It is possible that the added cost of a karyotype and the required culture will be difficult to justify in the future using arrays with more complete coverage.

Based on published data about the frequency of copy number variants in the population, ${ }^{24-27}$ there is a priori reason to be concerned about detection of variants of unknown clinical significance in prenatal studies. Our experience with over 1,500 blood analyses provided us with extensive data regarding which clones frequently detected regions of copy number variation. Our database of previously analyzed samples as well as public databases (such as http://projects.tcag.ca/variation) were researched in order to provide insight into the degree of variation detected by particular clones. With subsequent versions of the array, clones found to detect highly variant regions have been replaced. In addition, experience with blood analyses indicated that variants detected by a single clone, which were most frequent in telomeric regions, were usually present in an apparently healthy parent. This experience was generally confirmed in the prenatal studies. This leads us to recommend that blood be collected from both parents at or near the time of the prenatal procedure so that parental data can be available rapidly and prior to releasing an interpretation to the family. These will usually confirm that a CNV detected in a fetal sample is also present in a healthy parent. Some CNVs are detected by both array and FISH, and some are detected by array but not FISH with the same clone, so parents must be tested accordingly. In general, we have found that gains detected by clones containing segmental duplications may not be discriminated by FISH, requiring that parental samples be analyzed by array$\mathrm{CGH}$. Although distinguishing truly benign CNVs from others that may cause mild phenotypes with incomplete penetrance is a complex problem, ${ }^{28}$ it is likely that parents can justifiably be reassured if a variant in a fetus is present in a healthy parent. Interpretations should also take into account the possibility of nonpaternity or other genetic mechanisms, such as segregation of alleles in the case of one parent having a deletion and the other a reciprocal duplication.

The testing of direct fetal material by array-CGH analysis gave more rapid results (average six days) than karyotype or array-CGH of cultured cells. Direct fetal material was obtained either from sufficient quantities of chorionic villi or amniocytes, or after WGA of DNA obtained from smaller quantities of uncultured chorionic villi or freshly spun (direct) amnio- cytes. A comparison of the array-CGH results obtained for WGA treated fetal cells and cultured fetal cells from 58 cases demonstrated no difference. Thus, these findings support the use of direct fetal material for array-CGH analysis, with analysis of cultured fetal cells only if direct results are inconclusive. Others have reported the feasibility of performing array-CGH with DNA isolated from as little as $1 \mathrm{~mL}$ of uncultured amniotic fluid without using $\mathrm{WGA}^{18}$ and such a strategy would be attractive both to avoid any theoretical bias of amplification and to shorten the time for obtaining results. WGA could be used in some fraction of cases where analysis of unamplified DNA failed.

With the introduction of any new genetic test, patient and provider education about the risks, benefits, and limitations of the testing are paramount. A detailed pretest genetic counseling session was required of all participants in this study. Couples were more likely to opt for array-CGH testing if there was an ultrasound or other abnormality detected in their current pregnancy, if an abnormality occurred in a previous pregnancy, or if they were inclined to desire maximum available genetic testing. A common reason for declining participation in the study was a perception that the disorders tested on the microarray were rare. Although individual disorders tested by the array are indeed rare, their combined incidence is significant. Based on the known incidence for these various conditions, we estimate that the combined live-birth incidence in the general population of known genetic syndromes and subtelomeric rearrangements detected by array-CGH as used in this study is at least 1:1000 and may be as high as 1:500. Because of spontaneous loss of abnormal pregnancies, the frequency of abnormality is likely to be greater depending on the timing of the procedure. The use of array-CGH to study postnatal samples of individuals suspected to have genetic syndromes provides insight into the ability of the array-CGH platform to detect abnormalities. In our series of 1,200 postnatal cases referred to our clinical laboratory for array-CGH testing for suspected genetic disorders, the overall detection rate of clinically relevant disorders was approximately $7.0 \%$. Significantly, nearly all of these cases had a previous chromosome analysis that was normal. A larger study of several thousand pregnancies would be needed to ascertain the rate of detection of micro-deletion or duplication syndromes by array-CGH that cannot be detected by conventional karyotype analysis in pregnancies with known anomalies as well as in pregnancies with risk factors such as advanced maternal age.

Our initial experience with the application of array-CGH in the clinical prenatal diagnostic setting has demonstrated the feasibility and accuracy of a molecular-based screening approach for genomic imbalances. This technique has the potential to be faster, more sensitive, and detect many more abnormalities than a conventional karyotype. There may be an immediate indication for this enhanced testing in fetuses with congenital anomalies detected by ultrasound. In addition, couples who desire as much genetic information about their pregnancies as possible and who are undergoing amniocentesis or CVS, may wish to be made aware of the option for an expanded level of testing. Although not raised as an issue during the course of this 
study, the potential also exists for couples to tailor the results of the array on an individual basis to exclude conditions for which they do not wish information in a prenatal setting.

Additional large-scale studies are required in order to determine whether array-CGH may eventually replace a karyotype in routine prenatal diagnosis and to determine a detection rate for submicroscopic genomic imbalances using array-CGH. From a cost perspective, it is likely that automation and high throughput methodology could make array-CGH on direct samples without culturing less costly than conventional karyotype analysis of cultured cells while detecting a greater percentage of all abnormalities. Moreover, emerging methodologies based on isolation of fetal cells from the maternal cervix or blood or on isolation of fetal DNA from maternal plasma without invasive procedures might eventually change the guidelines for conventional prenatal genetic diagnosis. ${ }^{29-32}$

\section{ACKNOWLEDGMENTS}

Trilochan Sahoo, Jiangzhen Li, Seema R Lalani, Sung Hae Kang, Ankita Patel, and Patricia Ward, under the overall supervision of Sau Wai Cheung, were responsible for the performance, interpretation, and reporting of the array-CGH; Daniela del Gaudio under the supervision of Benjamin Roa developed the whole genome amplification protocol; Benjamin Roa oversaw the maternal cell contamination studies. Craig Chinault contributed to the design and oversaw production of the arrays; Chad Shaw developed the statistical analysis tools for a-CGH interpretation; Christine Eng and Arthur Beaudet were responsible for the overall design and oversight of the clinical study; Sandra Darilek, Sallie McAdoo, Audrey Burke, Ignatia Van den Veyver and Christine Eng conducted patient recruitment and genetic counseling. Trilochan Sahoo, Ignatia Van den Veyver, Christine Eng, and Arthur Beaudet drafted and revised the manuscript; all authors reviewed, revised and accepted the final version. The authors acknowledge Drs. Joe Leigh Simpson and James Lupski of Baylor College of Medicine for helpful discussions. The authors also thank George Weissenberger, Zhishuo Ou, Ellen Brundage, Svetlana Yatsenko, and Lance Cooper of Baylor College of Medicine for research and technical assistance.

\section{References}

1. Caughey AB, WA, Gildengorin V, Kuppermann M. Assessment of demand for prenatal diagnostic testing using willingness to pay. Obstet Gynecol 2004;103:539-545.

2. Kuppermann MNM. Prenatal testing guidelines: time for a new approach. Gynecol Obstet Invest 2005;60:6-10.

3. Harris RA, Washington AE, Nease RF, Jr., Kuppermann M. Cost utility of prenatal diagnosis and the risk-based threshold. Lancet 2004;363:276-282.

4. Tepperberg J, Pettenati MJ, Rao PN, Lese CM, et al. Prenatal diagnosis using interphase fluorescence in situ hybridization (FISH): 2-year multi-center retrospective study and review of the literature. Prenat Diagn 2001;21:293-301.

5. Leung WC, Waters JJ, Chitty L. Prenatal diagnosis by rapid aneuploidy detection and karyotyping: a prospective study of the role of ultrasound in 1589 secondtrimester amniocenteses. Prenat Diagn 2004;24:790-795.

6. Evans MI, Henry GP, Miller WA, Bui TH, et al. International, collaborative assessment of 146,000 prenatal karyotypes: expected limitations if only chromosome- specific probes and fluorescent in-situ hybridization are used. Hum Reprod 1999;14: 1213-1216.

7. Ward BE, Gersen SL, Carelli MP, McGuire NM, et al. Rapid prenatal diagnosis of chromosomal aneuploidies by fluorescence in situ hybridization: clinical experience with 4,500 specimens. Am J Hum Genet 1993;52:854-865.

8. ACOG. Prenatal diagnosis of fetal chromosome abnormalities. In, ACOG Practice Bulletin Number 27 . 2001.

9. Kallioniemi A, Kallioniemi OP, Sudar D, Rutovitz D, et al. Comparative genomic hybridization for molecular cytogenetic analysis of solid tumors. Science 1992;258: 818-821.

10. Pinkel D, Segraves R, Sudar D, Clark S, et al. High resolution analysis of DNA copy number variation using comparative genomic hybridization to microarrays. Nat Genet 1998;20:207-211.

11. Cheung SW, Shaw CA, Yu W, Li J, et al. Development and validation of a CGH microarray for clinical cytogenetic diagnosis. Genet Med 2005;7:422-432.

12. Wells D, Sherlock JK, Handyside AH, Delhanty JD. Detailed chromosomal and molecular genetic analysis of single cells by whole genome amplification and comparative genomic hybridisation. Nucleic Acids Res 1999;27:1214-1218.

13. Wells D, Delhanty JD. Comprehensive chromosomal analysis of human preimplantation embryos using whole genome amplification and single cell comparative genomic hybridization. Mol Hum Reprod 2000;6:1055-1062.

14. Bejjani BA, Saleki R, Ballif BC, Rorem EA, et al. Use of targeted array-based CGH for the clinical diagnosis of chromosomal imbalance: is less more? Am J Med Genet 2005;134:259-267.

15. Wong A, Lese Martin C, Heretis K, Ruffalo T, et al. Detection and calibration of microdeletions and microduplications by array-based comparative genomic hybridization and its applicability to clinical genetic testing. Genet Med 2005;7:264-271.

16. Cai WW, Mao JH, Chow CW, Damani S, et al. Genome-wide detection of chromosomal imbalances in tumors using BAC microarrays. Nat Biotechnol 2002;20:393-396.

17. Rickman L, Fiegler H, Carter NP, Bobrow M. Prenatal diagnosis by array-CGH. Eur J Med Genet 2005;48:232-240.

18. Rickman L, Fiegler H, Shaw-Smith C, Nash R, et al. Prenatal detection of unbalanced chromosomal rearrangements by array-CGH. J Med Genet 2005.

19. Vissers LE, de Vries BB, Osoegawa K, Janssen IM, et al. Array-based comparative genomic hybridization for the genomewide detection of submicroscopic chromosomal abnormalities. Am J Hum Genet 2003;73:1261-1270.

20. Yu W, Ballif BC, Kashork CD, Heilstedt HA, et al. Development of a comparative genomic hybridization microarray and demonstration of its utility with 25 wellcharacterized 1p36 deletions. Hum Mol Genet 2003;12:2145-2152.

21. Fox JE, Gloster ES, Mirchandani R. Trisomy 18 with Cantrell pentalogy in a stillborn infant. Am J Med Genet 1988;31:391-394.

22. Miura S, Miura K, Masuzaki H, Miyake N, et al. Microarray comparative genomic hybridization (CGH)-based prenatal diagnosis for chromosome abnormalities using cell-free fetal DNA in amniotic fluid. J Hum Genet 2006;51:412-417.

23. Le Caignec C, Spits C, Sermon K, De Rycke M, et al. Single-cell chromosomal imbalances detection by array CGH. Nucleic Acids Res 2006;34:e68.

24. Sharp AJ, Locke DP, McGrath SD, Cheng Z, et al. Segmental duplications and copy-number variation in the human genome. Am J Hum Genet 2005;77:78-88.

25. Sebat J, Lakshmi B, Troge J, Alexander J, et al. Large-scale copy number polymorphism in the human genome. Science 2004;305:525-528.

26. Iafrate AJ, Feuk L, Rivera MN, Listewnik ML, et al. Detection of large-scale variation in the human genome. Nat Genet 2004;36:949-951.

27. Conrad DF, Andrews TD, Carter NP, Hurles ME, et al. A high-resolution survey of deletion polymorphism in the human genome. Nat Genet 2005.

28. Rosenberg C, Knijnenburg J, Bakker E, Vianna-Morgante A, et al. Array-CGH detection of micro rearrangements in mentally retarded individuals: Clinical significance of imbalances present both in affected children and normal parents. J Med Genet 2005.

29. Adinolfi M, Sherlock J, Tutschek B, Halder A, et al. Detection of fetal cells in transcervical samples and prenatal diagnosis of chromosomal abnormalities. Prenat Diagn 1995;15:943-949.

30. Larrabee PB, Johnson KL, Pestova E, Lucas M, et al. Microarray analysis of cell-free fetal DNA in amniotic fluid: a prenatal molecular karyotype. Am J Hum Genet 2004;75:485-491.

31. Katz-Jaffe MG, Mantzaris D, Cram DS. DNA identification of fetal cells isolated from cervical mucus: potential for early non-invasive prenatal diagnosis. BJOG 2005; 112:595-600.

32. Bianchi DW. Circulating fetal DNA: its origin and diagnostic potential-a review. Placenta 2004;25:A93-A101. 